\title{
Detection of Electrophysiological Activity of Amygdala during Anesthesia Using Stereo-EEG: A Preliminary Research in Anesthetized Epileptic Patients
}

\author{
Tao Liang, ${ }^{1}$ Fan $W u^{2}$ Yongxing Sun $\mathbb{D}^{1},{ }^{1}$ and Baoguo Wang $\mathbb{D}^{1}$ \\ ${ }^{1}$ Department of Anesthesiology, Sanbo Brain Hospital, Capital Medical University, Beijing, China \\ ${ }^{2}$ Department of Anesthesiology, Affiliated Hospital of Inner Mongolia Medical University, Inner Mongolia, China \\ Correspondence should be addressed to Baoguo Wang; wbgsbnk@163.com
}

Received 10 March 2020; Revised 13 September 2020; Accepted 19 September 2020; Published 9 October 2020

Academic Editor: Stavros Baloyannis

Copyright (c) 2020 Tao Liang et al. This is an open access article distributed under the Creative Commons Attribution License, which permits unrestricted use, distribution, and reproduction in any medium, provided the original work is properly cited.

\begin{abstract}
Recent studies of anesthesia mechanisms have focused on neuronal network and functional connectivity. The stereoelectroencephalography (SEEG) recordings provide appropriate temporal and spatial resolution to study whole-brain dynamics; however, the feasibility to detect subcortical signals during anesthesia still needs to be studied with clinical evidence. Here, we focus on the amygdala to investigate if SEEG can be used to detect cortical and subcortical electrophysiological activity in anesthetized epileptic patients. Therefore, we present direct evidence in humans that SEEG indeed can be used to record cortical and subcortical electrophysiological activity during anesthesia. The study was carried out in propofol-anesthetized five epileptic patients. The electrophysiology activity of the amygdala and other cortical areas from anesthesia to the recovery of consciousness was investigated using stereo-EEG (SEEG). Results indicated that with the decrease of propofol concentration, power spectral density (PSD) in the delta band of the amygdala significantly decreased. When it was close to recovery, the correlation between the amygdala and ipsilateral temporal lobe significantly decreased followed by a considerable increase when awake. The findings of the current study suggest SEEG as an effective tool for providing direct evidence of the anesthesia mechanism.
\end{abstract}

\section{Introduction}

Recent studies of anesthesia mechanisms have focused on neuronal network and functional connectivity $[1,2]$. Anesthetic agents comprise a wide variety of molecules acting on numerous receptors, channels, and other protein targets in the body. At a small dose, anesthetics first suppress thinking, focused attention, and working memory. As the dose is increased, consciousness and voluntary responsiveness begin to fade. Functional neuroimaging by now has become a principal tool to study the neural correlates of consciousness [3]. In most studies, functional magnetic resonance imaging (fMRI) and electroencephalography (EEG) have been used to build neuronal networks $[4,5]$. However, direct observation of the brain deeper structure activity with EEG or fMRI measures is difficult $[6,7]$. Stereo-EEG (SEEG) is used to determine the localization of the epileptic focus before sur- gery in pharmaco-resistant epileptic patients [8]. Under the guidance of navigation, the electrode is implanted into the brain to directly record the electrical activity of the area. There are multiple contacts on the electrode; thus, they can be used for recording the cortical and subcortical electrophysiological activity accurately [9]. This precise process needs to be finished under general anesthesia. Therefore, SEEG provides us with a powerful tool to study neuronal brain dynamics with high temporal and good spatial resolution [10].

Being a less invasive and effective alternative investigative tool, SEEG is used for recording the seizures with a threedimensional analysis of the epileptic zone [11]. It has been widely used for the localization of the epileptic zones in different types of epilepsies. SEEG electrodes are generally preferred over ECoG grids when the lateralization of the seizures is unknown or is expected to be in deeper brain structures, 
such as the insula or hippocampus [12]. A recent published systemic analysis has shown that SEEG efficiently identified epileptic zones in about $92 \%$ of the patients who underwent SEEG before surgery [13]. Similarly, SEEG has been used effectively and successfully for the investigation of various types of epilepsies including temporal lobe epilepsy, extratemporal epilepsy, insular epilepsy, and various other types as summarized in a recently published review [14]. SEEG provides sparser coverage spanning more, bilateral brain regions including deeper structures. SEEG avoids unnecessary craniotomies and the associated morbidity, hospital stay, and costs [12].

Despite its wider implications in epileptic investigations, the feasibility of SEEG for the detection of subcortical signals during anesthesia still needs to be studied with clinical evidence. In this work, with the help of SEEG, we describe the electrophysiology activity of the amygdala and other cortical areas from anesthesia to recovery of consciousness (ROC) in epileptic patients and try to verify the feasibility of SEEG as an anesthesia research method.

\section{Materials and Methods}

2.1. Participants. This study was approved by the Ethics committee of Sanbo Brain Hospital, Capital Medical University (SBNK-YJYS-2019-009-01), and was registered in the Chinese Clinical Trial Registry (registration number: ChiCTR2000029067). All participants provided their written informed consent before the experiments. The whole experimental process was carried out according to the Declaration of Helsinki.

\subsection{Inclusion and Exclusion Criteria}

2.2.1. Inclusion Criteria. Following inclusion criteria was followed by the inclusion of the patients in this study: (1) patients with intractable epilepsy and who needed SEEG electrode implantation, (2) both male and female patients in 1840 -year age range, (3) grade I or II patients as classified by the American Society of Anesthesiologists (ASA) without serious systemic diseases (such as heart, lung, liver, and renal failure), and (4) patients capable of signing informed consent.

2.2.2. Exclusion Criteria. The following criteria as used for excluding the patients from the study: (1) patients with a history of anesthesia within six months; (2) breastfeeding or pregnant female patients; (3) patients participate in other clinical trials in the last four weeks; (4) patients using sleeping pills and analgesics for a long time or alcoholic patients; (5) extremely anxious, panicked patients having communication difficulties.

\section{Experimental Procedures}

3.1. Experimental Design and Procedure. In this study, the electrophysiological activity of the amygdala was observed from anesthesia to recovery. Total intravenous anesthesia (TIVA) was used for all patients. Propofol was administered as a target-controlled infusion (TCI) $(3.5 \mu \mathrm{g} / \mathrm{mL}$ of plasma) based on the pharmacokinetic model by Marsh et al. [15].
When all electrodes had been implanted, patients were transported to the Post Anesthesia Care Unit (PACU). SEEG activity from anesthesia to recovery was recorded in PACU. The following time periods were marked accurately: (A) propofol 3.5-3.0 $\mu \mathrm{g} / \mathrm{mL}$, (B) propofol 3.0-2.5 $\mu \mathrm{g} / \mathrm{mL}$, (C) propofol $2.5-2.0 \mu \mathrm{g} / \mathrm{mL}$, (D) propofol $2.0-1.5 \mu \mathrm{g} / \mathrm{mL}$, and (E) $1.5 \mu \mathrm{g} / \mathrm{mL}$-ROC. The experiment procedure is shown in Figure 1. The average period was $101 \mathrm{~s}, 123 \mathrm{~s}, 244 \mathrm{~s}, 644 \mathrm{~s}$, and $185 \mathrm{~s}$ for investigating $\mathrm{A}, \mathrm{B}, \mathrm{C}, \mathrm{D}$, and $\mathrm{E}$, respectively, as shown in Table S5.

3.2. EEG Recordings. Electrodes were implanted in five patients included in the study. The total number of electrodes for each patient was as follows: $y q y=15 ; w l=10 ; \mathrm{zyk}=15$; $\mathrm{zlx}=11$; and $\mathrm{wy}=13$. Multiple electrodes were implanted in each patient for observing the occurrence of epilepsy. However, we only focused on the amygdala and ipsilateral temporal lobe. Exact locations of the implanted electrodes are given in Table S1 of the Supplementary data file. SEEG activity was recorded from platinum-iridium semiflexible multicontact intracerebral electrodes, with a maximum of 16 contacts per electrode (Huake Hengsheng, China). The implantation of electrodes was guided by the navigation machine which was up to $0.2 \mathrm{~mm}$. The basic parameters of these implants are given in Figure S1 and Table S2 of the Supplementary data file. Each electrode contained different sites which are shown in Table S3. The distance between the recording sites was $1.5 \mathrm{~mm}$. As mentioned earlier, we only focused on the amygdala and ipsilateral temporal lobe; thus, many channels were excluded as shown in Table S4, and the details are given in the supplementary data file. EEG signals were collected using the Nicolet One EEG-64 device (Nicolet Corp., USA) with a sampling frequency of $1024 \mathrm{~Hz}$. The signals were band-passed at $1-55 \mathrm{~Hz}$ to avoid baseline drift and high-frequency noise. Signals were excluded from the analysis of those contacts that were located in (i) white matter (as assessed by MRI), (ii) epileptogenic zone (as confirmed by postsurgical assessment), (iii) over regions of documented alterations of the cortical tissue, and (iv) exhibited spontaneously or evoked epileptiform activity during recovery from anesthesia. The data were imported into Matlab (2016b) in ASCII format for further analysis.

3.3. EEG Analysis. Data analysis was performed with Brainstorm [16], which is documented and freely available for download online under the GNU general public license (http://neuroimage.usc.edu/brainstorm).

3.3.1. Power Spectrum Analysis. This process is aimed at evaluating the power of EEG signals at different frequencies, using Welch's method. Signals were split in overlapping windows of a given length, and the Fourier Transform (FFT) of each of these short segments and the average power of the FFT coefficients were calculated for all the overlapping windows [17]. The window length used for the splitting of the signal was 10 seconds, and the window overlap ratio was $50 \%$. The result was grouped in frequency bands: delta $2 \sim 4 \mathrm{~Hz}$, theta $5 \sim 7 \mathrm{~Hz}$, alpha $8 \sim 12 \mathrm{~Hz}$, and beta $15 \sim 29 \mathrm{~Hz}$. 


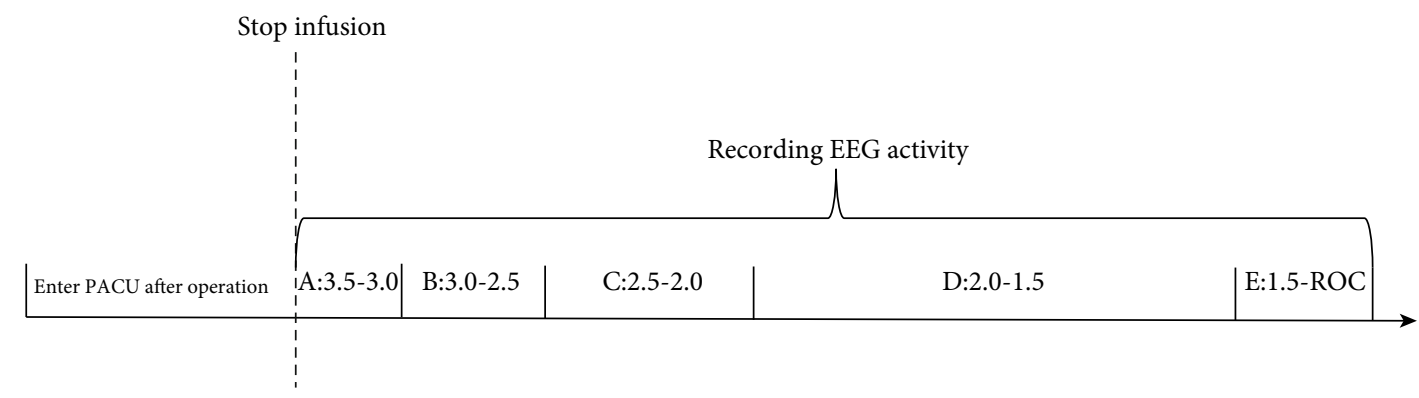

FIGURE 1: Schematic representation of the experimental procedure.

3.3.2. Time-Frequency Analysis. In this study, the wavelet transform was used to decompose EEG data to finish timefrequency analysis and to observe the changes of amygdala EEG in the process of anesthesia recovery. The purpose of this procedure was to observe the changes of different frequencies in the time domain during anesthesia to recovery. Time-frequency decomposition was computed based on the convolution of the signal with a series of complex Morlet wavelets. Wavelets had a variable resolution in time and frequency, while designing the wavelet, a trade-off between temporal and spectral resolution was decided. Therefore, in this study, the central frequency of $1 \mathrm{~Hz}$ and time resolution (FWHM) of $3 \mathrm{~s}$ were set [18]. The results were presented by the power of the wavelet coefficients.

3.3.3. EEG Correlation Analysis. The correlation is the basic approach to show the dependence or association among two random EEG signals [19]. The implantation plan of five patients involved both amygdala and the ipsilateral temporal lobe. Therefore, the correlation of the amygdala and temporal lobe in different periods was calculated in this study.

\section{Statistical Analysis}

Statistical analysis was performed using Matlab (2016b). The power spectral data from different electrodes of the amygdala were analyzed by one-way ANOVA followed by Tukey's honestly significant difference post hoc test. A paired sample $t$-test was applied to analyze the correlation at different times. A $p$ value of $<0.05$ was considered statistically significant.

\section{Result}

5.1. General Information. Five epilepsy Asian patients (three men and two women), aged in 19-31 years (mean of 24.6 years) and weighing $68-90 \mathrm{~kg}$ (mean of $78.4 \mathrm{~kg}$ ), were enrolled. The time for operation was $1.5-3 \mathrm{~h}$ (mean of $2.2 \mathrm{~h}$ ), time for recovery of consciousness was 20-37 min (mean of $26.8 \mathrm{~min}$ ), and fluid of crystal and colloid were $1940 \mathrm{~mL}$ and $600 \mathrm{~mL}$, respectively. The ranges for fluid of crystal and colloid were 1200 2700 mL and 500 1000 mL, respectively. All of the locations of electrodes are shown in Table 1. Multiple electrodes were implanted in each patient for observing the occurrence of epilepsy. However, we only focused on the amygdala and ipsilateral temporal lobe in this study. The illustration of the method of implant surgery is shown in Figure S2. The representative locations of the implanted electrodes are shown in Figure S3. For the correlation of the amygdala and temporal lobe, the middle temporal gyrus of the temporal lobe was investigated. In the case of the amygdala, its location was determined according to the postoperative imaging data and was considered as a whole.

5.2. Changes in the Overall PSD of the Amygdala. We evaluated the PSD of the amygdala in the frequency domain, and then, from the perspective of the time domain, we tried to demonstrate the correlation between them. To investigate the PSD of amygdale from anesthesia to recovery, EEG powers at each frequency band were calculated. It was found that when anesthesia was close to recovery, the PSD of the delta band decreased (Figure 2). One-way ANOVA analysis showed significant difference at delta band $(F=10.83$ and $p$ $<0.001)$, but not at theta $(F=0.42$ and $p=0.792)$, alpha $(F=3.36$ and $p=0.124)$, and beta $(F=0.84$ and $p=0.513)$ bands (Table S6).

5.3. Time-Frequency Analysis. Time-frequency analysis was conducted to demonstrate any change in the amygdala during the stable anesthesia. In the time-frequency analysis, it can be seen that all of the data show some similarity in the whole process. With the decrease of propofol concentration in plasma, the power of the delta band was always the highest. The order was delta $>$ theta $>$ alpha $>$ beta. We found that this method can be well repeated (Figure 3 ).

5.4. Correlation of Amygdala and Temporal Lobe. Next, we sought to investigate the status of the correlation between the amygdala and the ipsilateral temporal lobe under anesthesia. The extent of correlation changes of different propofol concentrations is presented in Figure 4. A paired $t$-test was used to analyze the changes in the correlation among different periods. The following effects were observed: at first, the correlation was relatively high in the following time periods: (A) propofol, 13.5-3.0 $\mu \mathrm{g} / \mathrm{mL}$; (B) propofol, 3.0-2.5 $\mu \mathrm{g} / \mathrm{mL}$; and (C) propofol, $2.5-2.0 \mu \mathrm{g} / \mathrm{mL}$. When the concentration of propofol decreased to (D) $2.0-1.5 \mu \mathrm{g} / \mathrm{mL}$, a significant decline was observed. However, a significant increase as observed when the drug concentration further decreased as shown in Table S7. 
TABle 1: Basic information of electrodes locations ( ${ }^{*}$ In: insular lobe; Fr: frontal lobe; Pa: parietal lobe; Am: amygdale; Te: temporal lobe; Fu: fusiform gyrus; Oc: occipital lobe; Ci: cingulate gyrus; En: entorhinal cortex; Hi: hippocampus).

\begin{tabular}{|c|c|c|c|c|c|c|c|c|c|c|c|c|c|c|c|c|}
\hline \multirow{2}{*}{ ID } & \multirow{2}{*}{ Sex } & \multirow{2}{*}{$\begin{array}{c}\text { Age } \\
\text { (years) }\end{array}$} & \multirow{2}{*}{$\begin{array}{l}\text { Height } \\
(\mathrm{cm})\end{array}$} & \multirow{2}{*}{$\begin{array}{l}\text { Weight } \\
(\mathrm{kg})\end{array}$} & \multirow{2}{*}{$\begin{array}{l}\text { Surgery } \\
\text { time (h) }\end{array}$} & \multirow{2}{*}{$\begin{array}{l}\text { ROC } \\
(\min )\end{array}$} & \multirow{2}{*}{$\begin{array}{c}\text { Crystal } \\
(\mathrm{mL})\end{array}$} & \multirow{2}{*}{$\begin{array}{l}\text { Colloid } \\
\text { (mL) }\end{array}$} & \multicolumn{8}{|c|}{ Electrode location } \\
\hline & & & & & & & & & 1 & 2 & 3 & 4 & 5 & 6 & 7 & 8 \\
\hline $\mathrm{zlx}$ & Male & 19 & 175 & 75 & $1.5 \mathrm{~h}$ & 37 & 2100 & 500 & $\mathrm{Am}_{\mathrm{R}}$ & $\begin{array}{c}\mathrm{Te}_{-} \\
\mathrm{R}\end{array}$ & $\begin{array}{c}\mathrm{Fu}_{-} \\
\mathrm{R}\end{array}$ & $\begin{array}{c}\mathrm{Te}_{-} \\
\mathrm{R}\end{array}$ & $\begin{array}{c}\mathrm{Te}_{-} \\
\mathrm{R}\end{array}$ & $\begin{array}{c}\mathrm{Te}_{-} \\
\mathrm{R}\end{array}$ & $\begin{array}{c}\mathrm{Fr}_{-} \\
\mathrm{R}\end{array}$ & $\begin{array}{c}\mathrm{Fr}_{-} \\
\mathrm{R}\end{array}$ \\
\hline zyk & Male & 22 & 171 & 80 & $2 \mathrm{~h}$ & 20 & 2100 & 500 & $\underset{\mathrm{L}}{\mathrm{Am}_{-}}$ & $\begin{array}{c}\mathrm{Te}_{-} \\
\mathrm{L}\end{array}$ & $\underset{\mathrm{L}}{\mathrm{Hi}}$ & $\begin{array}{c}\mathrm{Te}_{-} \\
\mathrm{L}\end{array}$ & $\underset{\mathrm{L}}{\mathrm{Hi}}$ & $\begin{array}{c}\mathrm{Te}_{-} \\
\mathrm{L}\end{array}$ & $\begin{array}{c}\mathrm{Oc}_{-} \\
\mathrm{L}\end{array}$ & $\begin{array}{c}\text { Oc } \\
\_L\end{array}$ \\
\hline wy & Female & 31 & 176 & 68 & $2 \mathrm{~h}$ & 22 & 2700 & 500 & In_L & $\begin{array}{c}\mathrm{Fr}_{-} \\
\mathrm{L}\end{array}$ & In_L & $\begin{array}{c}\mathrm{Pa}_{-} \\
\mathrm{L}\end{array}$ & $\underset{\mathrm{L}}{\mathrm{Am}}$ & $\begin{array}{c}\mathrm{Te}_{-} \\
\mathrm{L}\end{array}$ & $\mathrm{In}_{\mathrm{L}}$ & $\begin{array}{c}\mathrm{Fr}_{-} \\
\mathrm{L}\end{array}$ \\
\hline yqy & Male & 23 & 185 & 90 & $2.5 \mathrm{~h}$ & 25 & 1600 & 1000 & $\underset{\mathrm{L}}{\mathrm{Am}}$ & $\begin{array}{c}\mathrm{Te}_{-} \\
\mathrm{L}\end{array}$ & $\underset{\mathrm{R}}{\mathrm{Am}_{-}}$ & $\begin{array}{c}\mathrm{Te}_{-} \\
\mathrm{R}\end{array}$ & $\begin{array}{c}\mathrm{Fu} \\
\_\mathrm{L}\end{array}$ & $\begin{array}{c}\text { Oc } \\
\_L\end{array}$ & $\underset{\mathrm{L}}{\mathrm{Fu}_{-}}$ & $\begin{array}{c}\mathrm{Te}_{-} \\
\mathrm{L}\end{array}$ \\
\hline wl & Female & 28 & 155 & 79 & $3 \mathrm{~h}$ & 30 & 1200 & 500 & $\underset{\mathrm{R}}{\mathrm{Am}_{-}}$ & $\begin{array}{c}\mathrm{Te}_{-} \\
\mathrm{R}\end{array}$ & $\begin{array}{c}\mathrm{En}_{-} \\
\mathrm{R}\end{array}$ & $\begin{array}{c}\mathrm{Te}_{-} \\
\mathrm{R}\end{array}$ & $\begin{array}{r}\mathrm{Ci} \\
\quad \mathrm{R}\end{array}$ & $\begin{array}{c}\mathrm{Te}_{-} \\
\mathrm{R}\end{array}$ & $\underset{\mathrm{R}}{\mathrm{In}}$ & $\begin{array}{c}\mathrm{Te}_{-} \\
\mathrm{R}\end{array}$ \\
\hline
\end{tabular}

\section{Discussion}

The main aim of the current study is to identify the feasibility of using SEEG to observe subcortical electrophysiological activities from anesthesia to recovery of consciousness directly. We observed that low-frequency activity (delta) of the amygdala was strong under anesthesia and decreased gradually when it was close to awake. A previous study showed that propofolinduced anesthesia reduces the episodic memory reconsolidation in humans [20]. We also found that the correlation between the amygdala and the ipsilateral temporal lobe was stable under anesthesia. When the propofol concentration was decreased to $2.0-1.5 \mu \mathrm{g} / \mathrm{mL}$, a significant decrease in the correlation was observed. Previous studies also suggest the suppression of the complexity of regions sparsely connected with largescale brain networks as a mechanism of propofol-induced alteration in oriented reactivity to stimulation [21]. When the drug concentration further decreased, a significant increase was observed. This can be related to the regulation of fear under light anesthesia [22-24]. Effective connectivity, its dynamics, and its directionality are altered by propofol [25].

The amygdala is an important part of the subcortical limbic system [26]. Previous studies used PET and fMRI to measure the amygdala activity during anesthesia [27, 28]. However, these studies did not measure the spatial resolution in real time. In contrast, we used SEEG to record EEG data to locate the target area in real time [29]. Moreover, the previous studies give a very localized information about SEEG [30], whereas we try to give a more deep insight into the SEEG activity of the amygdala during anesthesia. Previously published reports of electrophysiological changes of the amygdala were studies for Spontaneous Inflammatory PainAssociated Activation of Pain Networks [31], change throughout postnatal development [32], and emotion categories of complex visual stimuli [33]. However, these studies did not evaluate the electrophysiological changes of the amygdala during anesthesia in epileptic patients. Timefrequency analysis of this study also observed that there was no significant change in the amygdala of the five patients during the stable anesthesia.

In some studies, EEG was used to observe the changes in the brain under anesthesia $[34,35]$. The spatial resolution of
EEG is not precise enough. Moreover, fMRI is also used to indirectly measure the activity of neurons before and after anesthesia in some studies. The spatial resolution of this method is good; however, it cannot monitor dynamically in real time. Previously Li et al. [36] suggest the use of the Laplacian re-reference for preprocessing in studies of broadband gamma and low-frequency oscillatory activity in SEEG signals. This assists in the emerging and unique SEEG method for the exploration of neural dynamics across the entire human brain; however, it was not specific for targeted location. In this study, we use SEEG to record EEG data, which can accurately locate the target area under the guidance of the navigation robot and meet the time and space resolution simultaneously. However, this is an invasive operation that can only be used for patients with intractable epilepsy. All of the five patients involved the amygdala and the ipsilateral temporal lobe in this study. Therefore, in the choice of research method, we observe the PSD of the amygdala in the frequency domain firstly, and then, from the perspective of the time domain, we tried to observe the correlation between them. This method has been widely used in electrophysiology, but it is not the best technique for finding the connectivity matrices. Multiple studies have reported high rates of seizure freedom following SEEG localization to the temporal lobe, ranging from $72.7 \%$ to $80 \%[9,37]$. However, it still can provide valuable information when we deal with a few narrow-banded signals in the time domain. Finally, we observed the changes of the amygdala from anesthesia to recovery with time-frequency analysis in different patients.

Recently, many researchers have tried to use SEEG to study the brain from different perspectives [38, 39]. However, the application of SEEG in the research of the anesthesia mechanism is still rare. Besides, the feasibility of this method used in the research of the anesthesia mechanism needs to be further demonstrated. Therefore, we provide direct evidence for the repeatability in this study.

\section{Limitation}

First, the sample size of this study is limited. In future studies, we will enlarge the sample size to cover more brain areas. Secondly, this study mainly focuses on propofol, and for other 

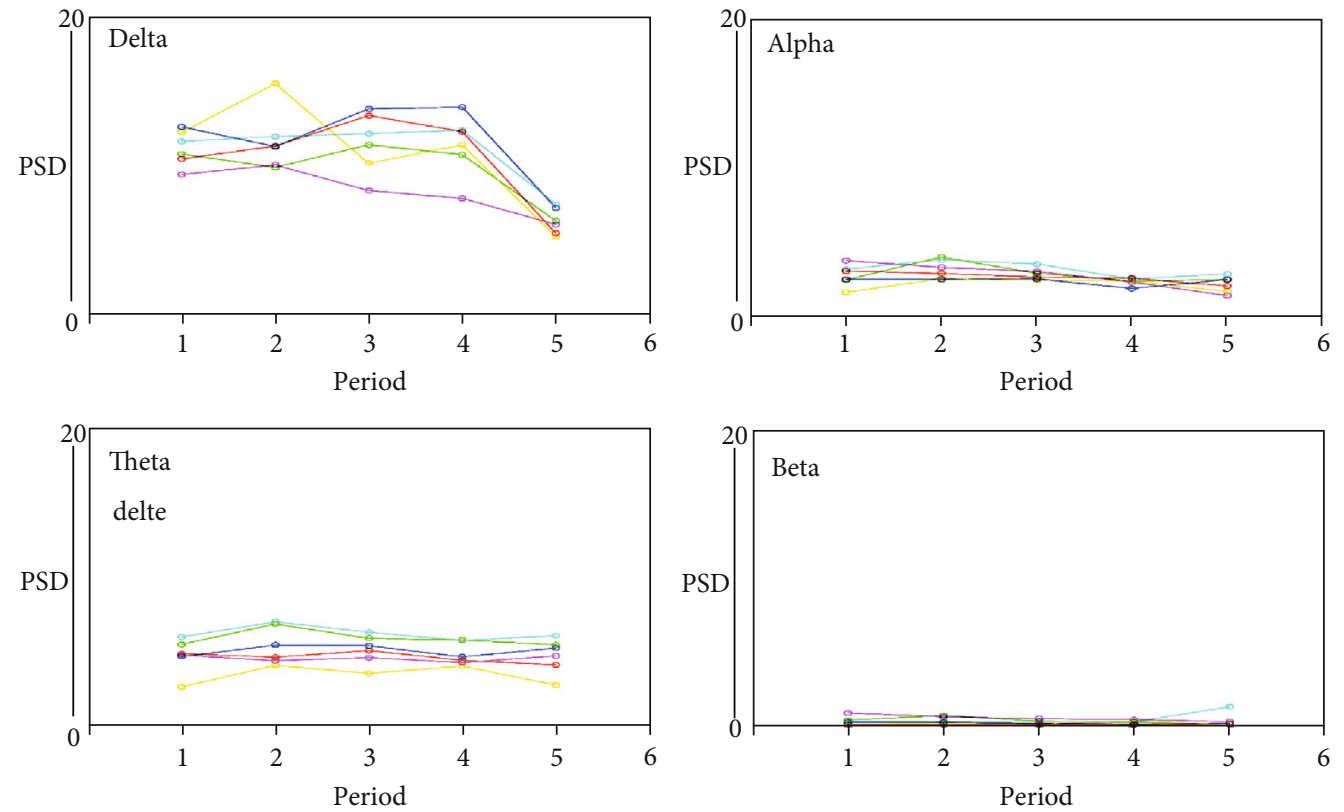

$$
\begin{aligned}
& -0 \text { wl } \\
& -0 \text { wy }
\end{aligned}
$$$$
\text { - } z i x
$$$$
\rightarrow \text { yqy }_{\mathrm{L}}
$$$$
- \text { yqy }_{\mathrm{R}}
$$

(a)
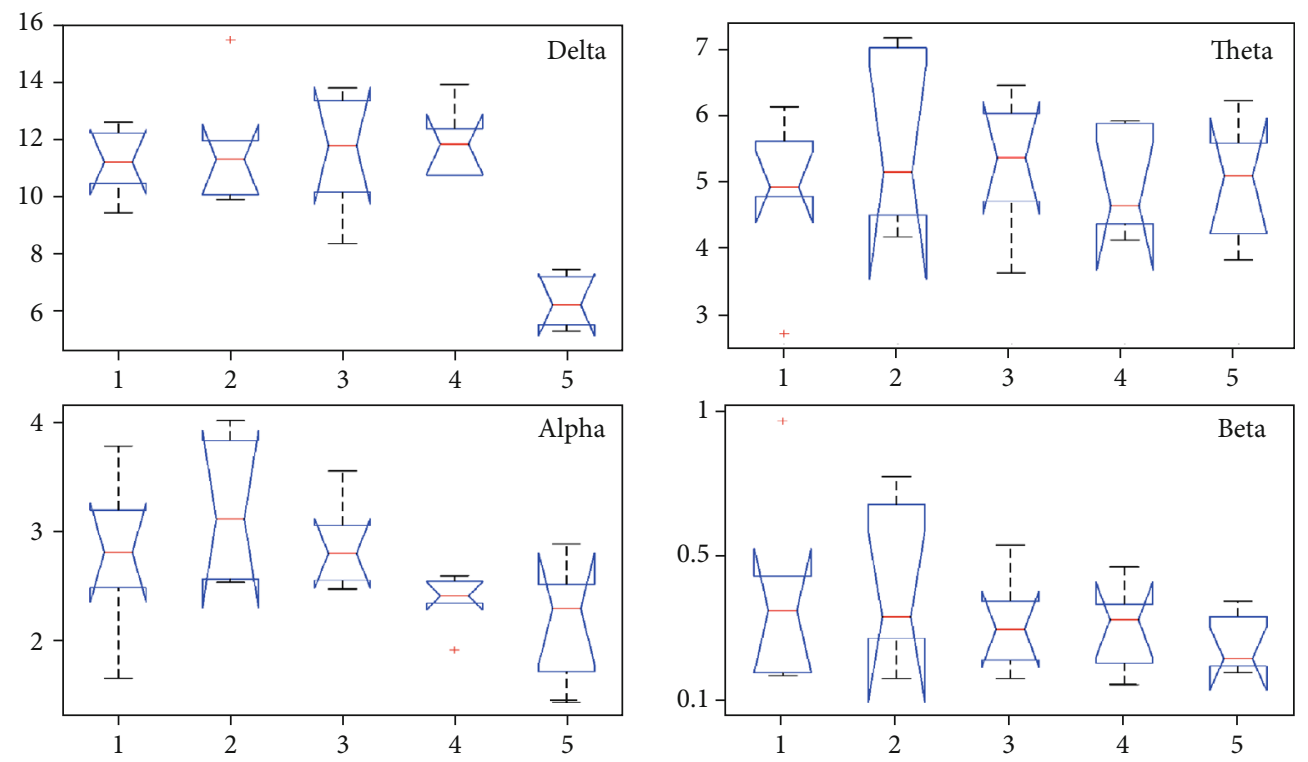

(b)

FIGURE 2: Evaluation of the PSD of the amygdala in the frequency domain where (a) delta band is the larger proportion in the whole process, and (b) there is no significant difference among theta, alpha, and beta bands. 

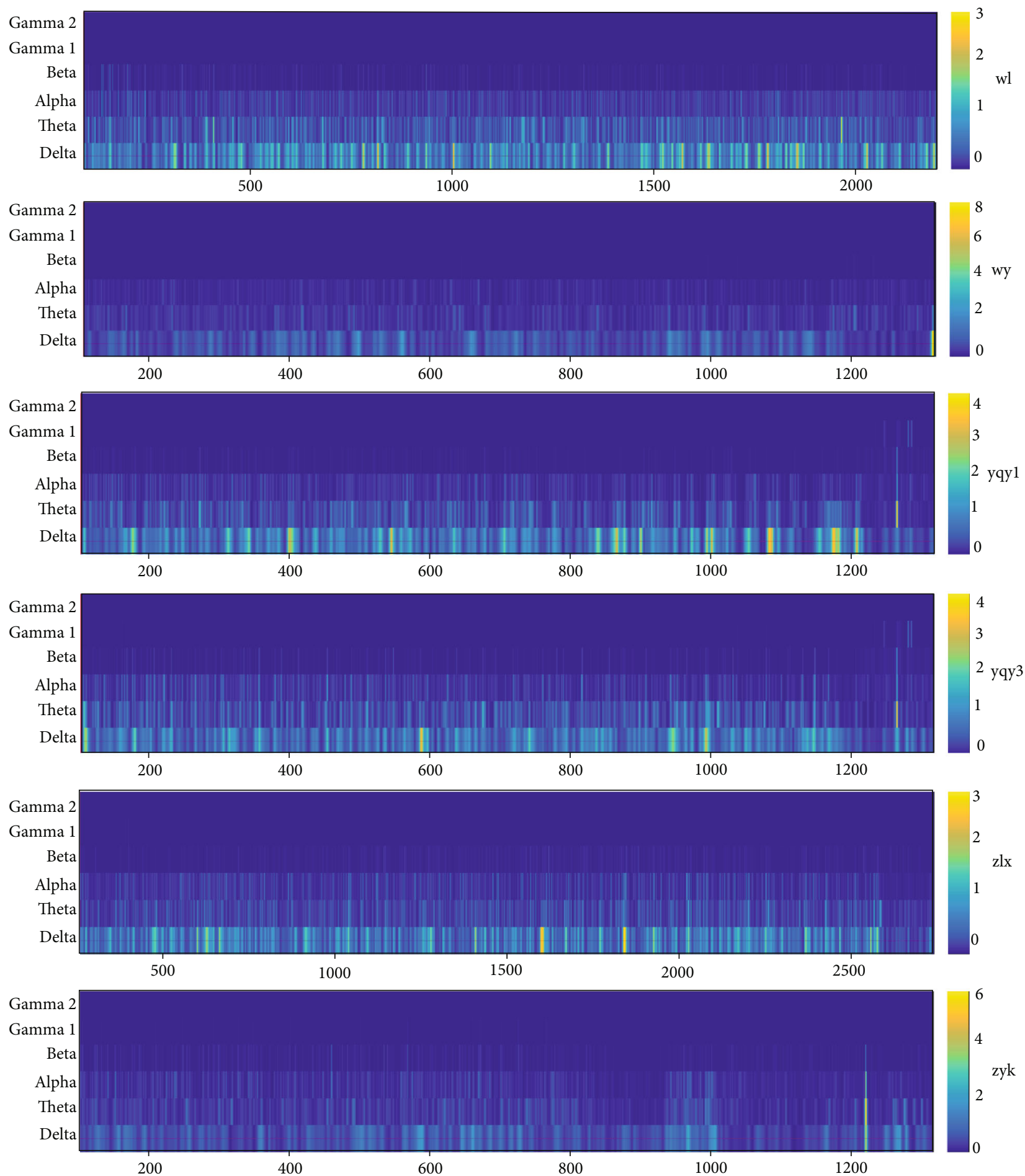

Figure 3: Time-frequency of amygdale indicating reproducibility of the method. 

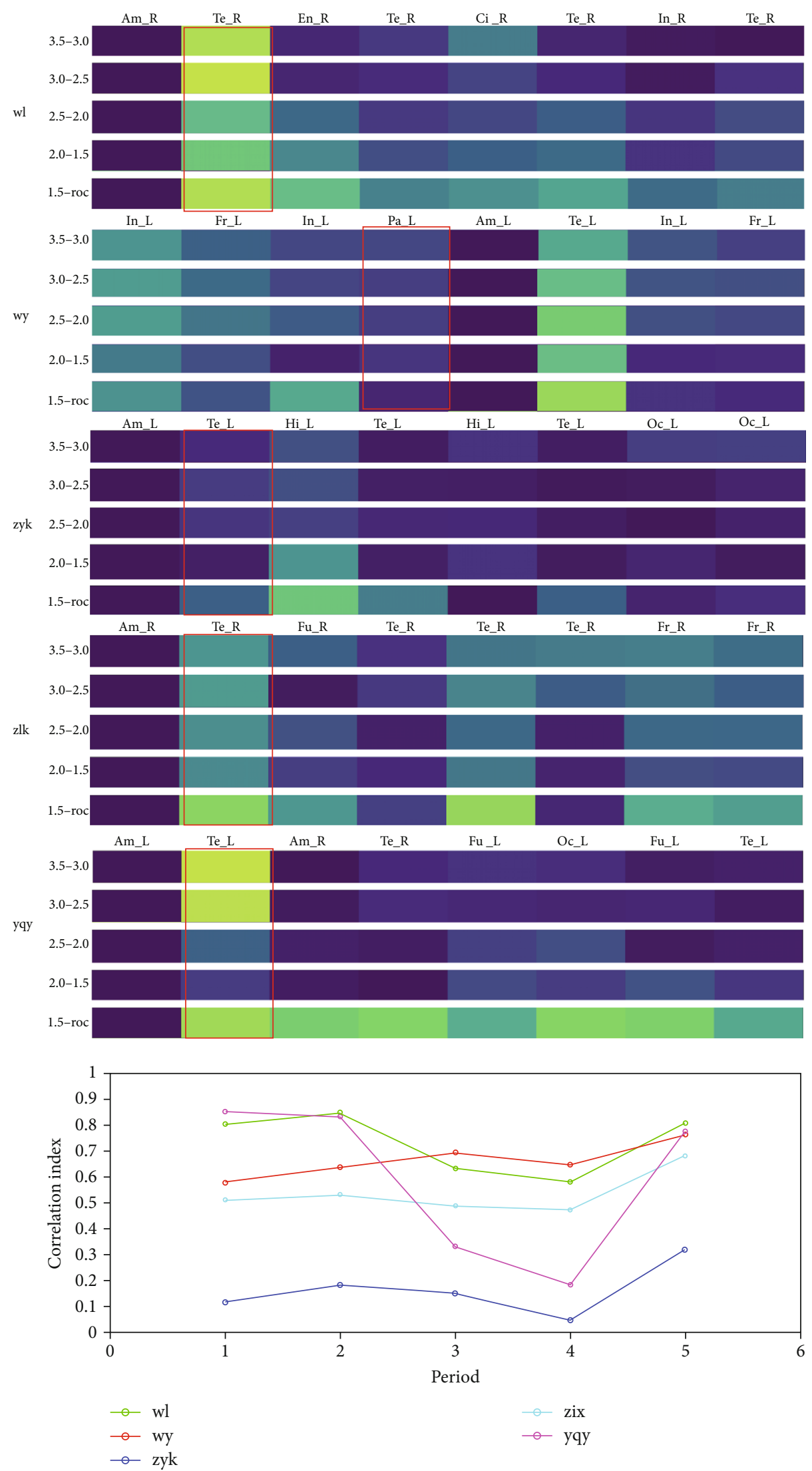

FIGURE 4: Correlation of amygdala and temporal lobe. 
drugs, further research is needed. Moreover, this is an exploratory research. The main purpose is to verify the feasibility of this method. Additionally, in the current study, resting oscillations may be quite abnormal in these patients and will need to design a strategy to overcome the abnormal oscillations. A large cohort study is still needed to further clarify the potential mechanisms of anesthesia.

\section{Conclusion}

Electrophysiology activity of the amygdala and other cortical areas from anesthesia to the recovery of consciousness was investigated in propofol-anesthetized five epileptic patients using stereo-EEG (SEEG). With the decrease of propofol concentration, PSD in the delta band of the amygdala decreased significantly. When it was close to awakening, the correlation between the amygdala and ipsilateral temporal lobe significantly decreased followed by a considerable increase when awake. Therefore, SEEG can be used to provide direct evidence for the study of the anesthesia mechanism.

\section{Data Availability}

There is no such data.

\section{Conflicts of Interest}

All the authors declare no conflict of interest.

\section{Acknowledgments}

All the authors acknowledge Sanbo Brain Hospital of Capital Medical University for the completion of this study. This work was funded by the Capital Medical Development Research Fund (2016-1-20410).

\section{Supplementary Materials}

In the supplementary materials, we present the following contents: Table S1, Table S2, Table S3, Table S4, Table S5, Table S6, Table S7, Figure S1, Figure S2, Figure S3, and the method of implant surgery. (Supplementary Materials)

\section{References}

[1] U. Lee and G. A. Mashour, "Role of network science in the study of anesthetic state transitions," Anesthesiology, vol. 129, no. 5, pp. 1029-1044, 2018.

[2] A. G. Hudetz and G. A. Mashour, "Disconnecting consciousness: is there a common anesthetic end-point?," Anesthesia and Analgesia, vol. 123, no. 5, pp. 1228-1240, 2016.

[3] V. Bonhomme, P. Boveroux, P. Hans et al., "Influence of anesthesia on cerebral blood flow, cerebral metabolic rate, and brain functional connectivity," Current Opinion in Anaesthesiology, vol. 24, no. 5, pp. 474-479, 2011.

[4] X. Liu, K. K. Lauer, B. D. Ward, S. J. Li, and A. G. Hudetz, "Differential effects of deep sedation with propofol on the specific and nonspecific thalamocortical Systems," Anesthesiology, vol. 118, no. 1, pp. 59-69, 2013.
[5] H. Kim, A. G. Hudetz, J. Lee, G. A. Mashour, U. C. Lee, and the ReCCognition Study Group, "Estimating the integrated information measure phi from high-density electroencephalography during states of consciousness in humans," Frontiers in Human Neuroscience, vol. 12, p. 42, 2018.

[6] A. Ranft, D. Golkowski, T. Kiel et al., "Neural correlates of sevoflurane-induced unconsciousness identified by simultaneous functional magnetic resonance imaging and electroencephalography," Anesthesiology, vol. 125, no. 5, pp. 861-872, 2016.

[7] D. Li, P. E. Vlisides, M. B. Kelz, M. S. Avidan, G. A. Mashour, and ReCCognition Study Group, "Dynamic cortical connectivity during general anesthesia in healthy volunteers," Anesthesiology, vol. 130, no. 6, pp. 870-884, 2019.

[8] F. Panzica, G. Varotto, F. Rotondi, R. Spreafico, and S. Franceschetti, "Identification of the epileptogenic zone from stereo-EEG signals: a connectivity-graph theory approach," Frontiers in Neurology, vol. 4, p. 175, 2013.

[9] M. Cossu, F. Cardinale, L. Castana et al., "Stereoelectroencephalography in the presurgical evaluation of focal epilepsy: a retrospective analysis of 215 procedures," Neurosurgery, vol. 57, no. 4, pp. 706-718, 2005.

[10] M. Bola, A. B. Barrett, A. Pigorini, L. Nobili, A. K. Seth, and A. Marchewka, "Loss of consciousness is related to hypercorrelated gamma-band activity in anesthetized macaques and sleeping humans," NeuroImage, vol. 167, pp. 130-142, 2018.

[11] R. S. Centeno, E. M. T. Yacubian, L. O. S. F. Caboclo, H. Carrete Júnior, and S. Cavalheiro, "Intracranial depth electrodes implantation in the era of image-guided surgery," Arquivos de Neuro-Psiquiatria, vol. 69, no. 4, pp. 693-698, 2011.

[12] C. Herff, D. J. Krusienski, and P. Kubben, "The potential of stereotactic-EEG for brain-computer interfaces: current progress and future directions," Frontiers in Neuroscience, vol. 14, p. 123, 2020.

[13] B. Garcia-Lorenzo, T. del Pino-Sedeño, R. Rocamora, J. E. López, P. Serrano-Aguilar, and M. M. Trujillo-Martín, "Stereoelectroencephalography for refractory epileptic patients considered for surgery: systematic review, meta-analysis, and economic evaluation," Neurosurgery, vol. 84, no. 2, pp. 326-338, 2019.

[14] B. E. Youngerman, F. A. Khan, and G. M. McKhann, "Stereoelectroencephalography in epilepsy, cognitive neurophysiology, and psychiatric disease: safety, efficacy, and place in therapy," Neuropsychiatric Disease and Treatment, vol. 15, pp. 1701-1716, 2019.

[15] B. Marsh, M. White, N. Morton, and G. N. C. Kenny, "Pharmacokinetic model driven infusion of propofol in children," British Journal of Anaesthesia, vol. 67, no. 1, pp. 41-48, 1991.

[16] F. Tadel, S. Baillet, J. C. Mosher, D. Pantazis, and R. M. Leahy, "Brainstorm: a user-friendly application for MEG/EEG analysis," Computational Intelligence and Neuroscience, vol. 2011, Article ID 879716, 13 pages, 2011.

[17] S. K. Mitra, Digital Signal Processing : A Computer-Based Approach, McGraw-Hill Education, 4th edition, 2013.

[18] R. Alazrai, H. Alwanni, Y. Baslan, N. Alnuman, and M. Daoud, "EEG-Based Brain-computer interface for decoding motor imagery tasks within the same hand using Choi-Williams time-frequency distribution," Sensors (Basel), vol. 17, no. 9, article 1937, 2017.

[19] F. Tadel, E. Bock, G. Niso et al., "MEG/EEG group analysis with brainstorm," Frontiers in Neuroscience, vol. 13, p. 76, 2019. 
[20] A. Galarza Vallejo, M. C. W. Kroes, E. Rey et al., "Propofolinduced deep sedation reduces emotional episodic memory reconsolidation in humans," Science Advances, vol. 5, no. 3, article eaav3801, 2019.

[21] I. Pappas, R. M. Adapa, D. K. Menon, and E. A. Stamatakis, "Brain network disintegration during sedation is mediated by the complexity of sparsely connected regions," NeuroImage, vol. 186, pp. 221-233, 2019.

[22] T. B. Lonsdorf, M. M. Menz, M. Andreatta et al., "Don't fear 'fear conditioning': methodological considerations for the design and analysis of studies on human fear acquisition, extinction, and return of fear," Neuroscience \& Biobehavioral Reviews, vol. 77, pp. 247-285, 2017.

[23] C. Trenado, N. Pedroarena-Leal, L. Cif, M. Nitsche, and D. Ruge, "Neural oscillatory correlates for conditioning and extinction of fear," Biomedicine, vol. 6, no. 2, p. 49, 2018.

[24] N. Karalis, C. Dejean, F. Chaudun et al., "4-Hz oscillations synchronize prefrontal-amygdala circuits during fear behavior," Nature Neuroscience, vol. 19, no. 4, pp. 605-612, 2016.

[25] U. Lee, S. W. Ku, G. J. Noh, S. H. Baek, B. M. Choi, and G. A. Mashour, "Disruption of frontal-parietal communication by ketamine, propofol, and sevoflurane," Anesthesiology, vol. 118, no. 6, pp. 1264-1275, 2013.

[26] S. B. E. Wolff, J. Gründemann, P. Tovote et al., “Amygdala interneuron subtypes control fear learning through disinhibition," Nature, vol. 509, no. 7501, pp. 453-458, 2014.

[27] M. T. Alkire, R. Gruver, J. Miller, J. R. McReynolds, E. L. Hahn, and L. Cahill, "Neuroimaging analysis of an anesthetic gas that blocks human emotional memory," Proceedings of the National Academy of Sciences of the United States of America, vol. 105, no. 5, pp. 1722-1727, 2008.

[28] K. O. Pryor and M. M. Berger, "The elusive promise of perioperative hyperoxia," British Journal of Anaesthesia, vol. 115, no. 3, pp. 344-346, 2015.

[29] R. R. Rozeske and C. Herry, "Neuronal coding mechanisms mediating fear behavior," Current Opinion in Neurobiology, vol. 52, pp. 60-64, 2018.

[30] J. Parvizi and S. Kastner, "Promises and limitations of human intracranial electroencephalography," Nature Neuroscience, vol. 21, no. 4, pp. 474-483, 2018.

[31] D. Arimura, K. Shinohara, Y. Takahashi et al., "Primary role of the amygdala in spontaneous inflammatory pain- associated activation of pain networks - a chemogenetic manganeseenhanced MRI approach," Frontiers in Neural Circuits, vol. 13, pp. 58-58, 2019.

[32] D. E. Ehrlich, S. J. Ryan, and D. G. Rainnie, "Postnatal development of electrophysiological properties of principal neurons in the rat basolateral amygdala," The Journal of Physiology, vol. 590, no. 19, pp. 4819-4838, 2012.

[33] H. Oya, H. Kawasaki, M. A. Howard III, and R. Adolphs, "Electrophysiological responses in the human amygdala discriminate emotion categories of complex visual stimuli," The Journal of Neuroscience, vol. 22, no. 21, pp. 95029512, 2002.

[34] M. Boly, R. Moran, M. Murphy et al., "Connectivity changes underlying spectral EEG changes during propofol-induced loss of consciousness," Journal of Neuroscience, vol. 32, no. 20, pp. 7082-7090, 2012.

[35] V.S. Hambrecht-Wiedbusch, D. Li, and G. A. Mashour, "Paradoxical emergence: administration of subanesthetic ketamine during isoflurane anesthesia induces burst suppression but accelerates recovery," Anesthesiology, vol. 126, no. 3, pp. 482494, 2017.

[36] G. Li, S. Jiang, S. E. Paraskevopoulou et al., "Optimal referencing for stereo-electroencephalographic (SEEG) recordings," NeuroImage, vol. 183, pp. 327-335, 2018.

[37] J. González-Martínez, J. Bulacio, S. Thompson et al., "Technique, results, and complications related to robot-assisted Stereoelectroencephalography," Neurosurgery, vol. 78, no. 2, pp. 169-180, 2016.

[38] G. Arnulfo, J. Hirvonen, L. Nobili, S. Palva, and J. M. Palva, "Phase and amplitude correlations in resting-state activity in human stereotactical EEG recordings," NeuroImage, vol. 112, pp. 114-127, 2015.

[39] M. M. Schartner, A. Pigorini, S. A. Gibbs et al., "Global and local complexity of intracranial EEG decreases during NREM sleep," Neuroscience of consciousness, vol. 2017, no. 1, article niw022, 2017. 